\title{
Defect energetics and impurity incorporation mechanisms at the arsenic-passivated $\mathrm{Si}(\mathbf{1 0 0})$ surface
}

\author{
M. Ramamoorthy, E. L. Briggs, and J. Bernholc \\ Department of Physics, North Carolina State University, Raleigh, North Carolina 27695-8202
}

(Received 10 November 1998)

\begin{abstract}
Theoretical calculations show that defect properties of the $\mathrm{Si}(100)$ and $\mathrm{Si}(100)$ :As surfaces are completely different. Large atomic relaxations around vacancies near the $\mathrm{Si}(100)$ surface cause chemical rebonding and defect healing that greatly lowers their formation energies. However, passivation of the surface by a monolayer of As induces substantial structural rigidity in the near-surface region. This reduces atomic relaxations and raises vacancy formation energies to high values, inhibiting vacancy mediated processes near the surface. The formation energies of silicon interstitials near the As-passivated surface are significantly lower than those of vacancies, which favors an interstitial mode of arsenic incorporation into the bulk during in diffusion. These results explain the observed uniformity of the $\mathrm{Si}(100)$ :As surface and the high level of electrical activation of in-diffused As. [S0163-1829(99)00835-8]
\end{abstract}

\section{INTRODUCTION}

Arsenic atoms adsorbed on the $\mathrm{Si}(100)$ surface readily incorporate into the surface layer upon annealing at moderate temperatures. ${ }^{1}$ This results in the formation of a monolayer of As atoms terminating the surface that completely passivates all surface dangling bonds and reduces the surface energy. ${ }^{2-5}$ During the epitaxial growth of Si and Si-Ge films, this impurity monolayer acts as a surfactant that segregates to the growing surface and assists in layer-by-layer growth of the semiconducting material. The process of surfactantmediated growth has been the subject of numerous investigations over the past decade and a number of distinct mechanisms have been proposed. ${ }^{6}$

A key ingredient that implicitly underlies all proposed explanations of the surfactant properties of As at $\mathrm{Si}(100)$ is the uniformity and stability of the terminating As monolayer. It is generally observed ${ }^{5,7}$ that the As-terminated surface, denoted in the following as $\mathrm{Si}(100)$ :As, is much more well ordered and defect free than the clean $\mathrm{Si}(100)$ surface, which is prone to having a significant number of surface divacancies and addimers. ${ }^{8,9}$ This is in contrast to the situation with many other elements that are capable of saturating all surface dangling bonds, but instead give rise to disordered or partially ordered surfaces. ${ }^{10,11}$ Indeed, in a comprehensive review of As passivation of $\mathrm{Si}$ and Ge surfaces, Bringans ${ }^{5}$ points out that As passivation is "more unique than it is first apparent.' The reasons causing the exceptional resistance of $\mathrm{Si}(100)$ :As to defect formation have not been understood, to the best of our knowledge.

Another issue that remains to be addressed is the mechanism of incorporation of adsorbed As atoms through the $\mathrm{Si}(100)$ surface into the bulk of the semiconducting material. It is well known that native point defects form complexes with impurities and facilitate their migration in bulk silicon. In the case of arsenic, systematic experimental and theoretical investigations have elucidated a number of defect-related phenomena in bulk silicon: vacancy and interstitial assisted diffusion mechanisms, ${ }^{12,13}$ anomalous diffusion, and formation of inactive complexes with vacancies at high doping levels, ${ }^{14-16}$ precipitation of interstitial phases, etc. ${ }^{17,18} \mathrm{It}$ might be expected that point defects also facilitate the pro- cess of diffusive incorporation of adsorbed As impurities.

However, relatively little is known about the interaction of impurities with point defects near the Si surfaces. For the clean $\mathrm{Si}(100)$ surface, attention has been restricted to defects in the surface layer or adlayer. ${ }^{8,9,19-22}$ The structure and formation energies of defects at and near the $\mathrm{Si}(100)$ :As are still unknown, to the best of our knowledge. Given that the sizes of Si-based devices are shrinking to the submicron range, the atomic layers near the surface will form an increasing fraction of the next generations of these devices. Thus, a detailed understanding of impurity configurations and migration mechanisms is of great importance in the simulation of device processing.

In this paper, we report a systematic theoretical investigation of defects at and near the $\mathrm{Si}(100)$ and $\mathrm{Si}(100)$ :As surfaces. The formation energies of both vacancies and interstitials near the clean surface are significantly lower than those in the bulk. In the case of vacancies, this is readily explained by the dramatic atomic rearrangements and chemical rebonding of neighboring $\mathrm{Si}$ atoms. However, at the $\mathrm{Si}(100)$ :As surface, the relaxations around vacancies are negligible. Therefore, Si neighbors of the vacancies remain undercoordinated, resulting in their formation energies being in the neighborhood of bulk values. On both surfaces, interstitials near the surface have reduced formation energies relative to the bulk, but their values at $\mathrm{Si}(100)$ :As are significantly larger than those near $\mathrm{Si}(100)$. The implications of these results on the mechanism of diffusive As incorporation through the surfaces is examined. While low-energy vacancy-assisted pathways of impurity incorporation are available on the clean $\mathrm{Si}(100)$ surface, at $\mathrm{Si}(100)$ :As the energetically favorable incorporation mechanisms involve interstitials. These results explain the observed stability of the Si(100):As surface against defect formation and shed light on the process of generation of interstitials in As-doped silicon.

\section{CALCULATiONS}

The total energies of various surface structures were calculated using the density-functional method. ${ }^{23}$ The local- 
density approximation was used for the exchange and correlation contribution. Atoms were represented using norm conserving pseudopotentials ${ }^{24}$ and electronic wave functions were calculated using a real-space multigrid-based approach. ${ }^{25}$ A real-space grid spacing of 0.6 a.u., corresponding to a plane-wave cutoff of $13.8 \mathrm{Ry}$, gave converged results. Our results for the bulk Si lattice constant and the structure of the $(2 \times 1) \mathrm{Si}(100)$ surface are in good agreement with experiment and earlier theoretical work. ${ }^{8,26}$

The supercell approach was used to calculate the properties of point defects. Defects on the $(2 \times 1)$ surface were studied using a $(4 \times 4)$ surface unit cell, with a vacuum layer corresponding to six layers of bulk silicon. The results were tested for convergence with respect to slab thickness by carrying out initial calculations with six layer slabs and then refining the key results with ten layer slabs. Similarly, they were tested for $k$-point convergence using the $\Gamma k$ point and the $(0.25,0.25,0)$ special $k$ point. These convergence tests indicate that the results are accurate to within $0.2-0.3 \mathrm{eV}$.

\section{NATIVE DEFECTS AT THE CLEAN (100) SURFACE}

In perfect bulk silicon, all the atoms are fourfold coordinated. Thus, when a $\mathrm{Si}$ atom is removed, the neighbors of the vacancy are left threefold coordinated. On the other hand, when an excess $\mathrm{Si}$ atom is placed at an interstitial position, the chemical configuration of atoms in the vicinity of the defect is significantly different from the tetrahedral bonding found in the ideal, bulk material. The structural relaxations around a point defect are strongly constrained and there is little relief of the strain in the system. It is thus not very surprising that the equilibrium formation energies of the vacancy and the interstitial have high magnitudes of 3.9 and $3.3 \mathrm{eV}$, respectively, consistent with their small concentration levels, even at relatively high temperatures.

At the $(2 \times 1)$ reconstructed $\mathrm{Si}(100)$ surface, the atoms are only threefold coordinated and are arranged in rows of dimers. ${ }^{8,26}$ This gives rise to a high density of surfacedangling bonds, reflected in the alternate, ordered buckling of the surface dimers. Given the reduced coordination of the surface atoms and significant structural corrugation found at this surface, it may be expected that a number of novel lowenergy defect structures might exist. Indeed, we will show in the following that dramatic atomic rearrangements occur around near-surface defects, which result in significant chemical rebonding of the neighboring $\mathrm{Si}$ atoms. They reduce the number of undercoordinated $\mathrm{Si}$ atoms and, in turn, reduce the formation energies of defects compared to the bulk.

We first present the results for the structural configurations of $\mathrm{Si}$ adatoms, addimers, and surface divacancies, which have also been the subject of earlier theoretical and experimental investigations. We find the equilibrium position of the $\mathrm{Si}$ adatom to be at the side of a dimer row, where it is in between two neighboring Si dimers of one row, bonded to two top layer $\mathrm{Si}$ atoms and one second layer $\mathrm{Si}$ atom. The $\mathrm{Si}$ addimer prefers to be adsorbed on top of a row of dimers, placed in between two neighboring dimers. The two orientations, parallel and perpendicular to the dimer rows, differ in energy by only $0.1 \mathrm{eV}$. The formation energy of the Si addimer is only about $0.5 \mathrm{eV},{ }^{27}$ because its bonding configura- (a)

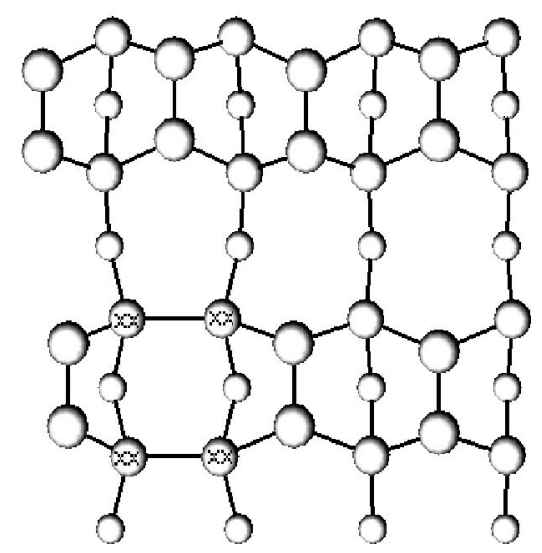

(b)

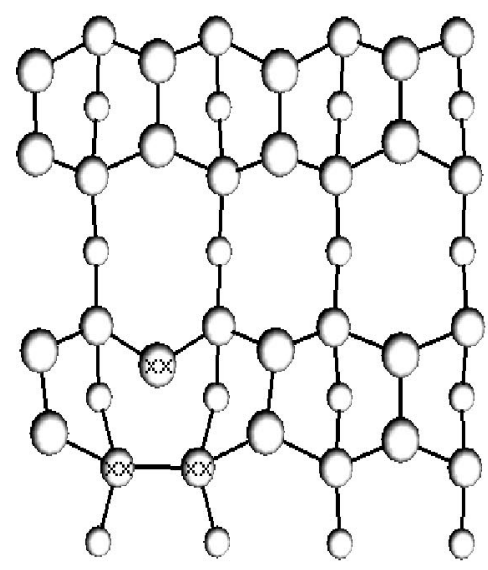

(c)

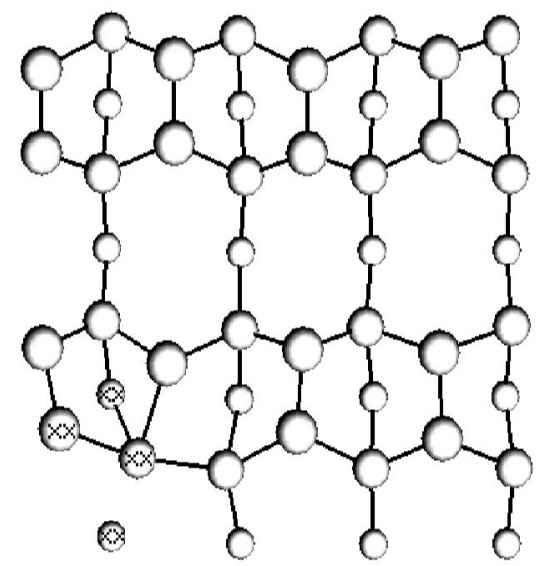

FIG. 1. Top view of the relaxed structures of vacancies at the $\mathrm{Si}(100)$ surface. The Si neighbors of the vacancies are marked with " $x x$." (a) surface divacancy; (b) first layer vacancy; (c) second layer vacancy.

tion is rather similar to that of a surface Si dimer. A defect that has a comparably low formation energy of about $0.5 \mathrm{eV}$ is the surface divacancy, shown in Fig. 1(a). The four subsurface $\mathrm{Si}$ neighbors of the divacancy move towards each other to form a pair of strained dimers, again quite similar to the dimers of the clean surface. The Si-Si bonds of the strained dimers in the second layer are about 2.6-2.7 $\AA$, $20 \%$ larger than those of Si dimers on the clean $\mathrm{Si}(100)$ surface. These results are in excellent agreement with earlier theoretical investigations ${ }^{19,21,20}$ and are also consistent with scanning tunneling microscopy (STM) observations. ${ }^{9,22}$

Having demonstrated the accuracy of our results by com- 


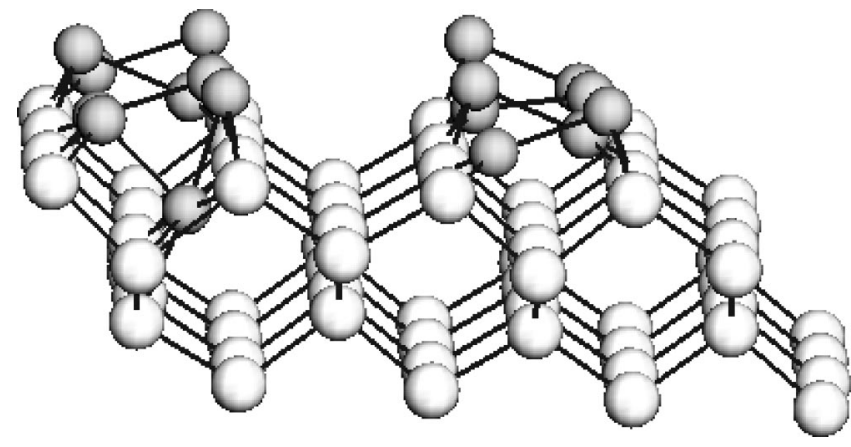

FIG. 2. Side view of the relaxed structure of the lowest energy $\mathrm{Si}$ interstitial configuration near the $\mathrm{Si}(100)$ surface. The surface $\mathrm{Si}$ atoms and the $\mathrm{Si}$ interstitial are shaded for clarity.

parison to previously published work, we present a detailed analysis of vacancies and interstitials at and below the surface. Vacancies in the first and second layers have formation energies of about $0.9 \mathrm{eV}$, which are still much lower than the value of $3.9 \mathrm{eV}$ in the bulk. In Figs. 1(b) and 1(c), the structural relaxations around these low-energy vacancy configurations are depicted. The large relaxations of the nearestneighbor $\mathrm{Si}$ atoms give rise to significant chemical rebonding. For the vacancy in the surface layer, one surface $\mathrm{Si}$ neighbor is left twofold coordinated. However, the two $\mathrm{Si}$ neighbors in the second layer move towards each other by about $0.5 \AA$ each, forming a $2.5 \AA$ bond. For the second layer vacancy, one of the surface Si neighbors moves so far as to almost occupy the position of the missing $\mathrm{Si}$ atom, forming five Si bonds, four of which are in the range 2.4-2.5 $\AA$ and the fifth is $2.6 \AA$. These bonds are all within 10-20\% of the $2.35 \AA$ value of the $\mathrm{Si}-\mathrm{Si}$ bond in bulk Si. Therefore, the $\mathrm{Si}(100)$ surface reveals tremendous flexibility in accommodating large atomic rearrangements around vacancies, which occur to reduce the degree of undercoordination of the neighboring $\mathrm{Si}$ atoms.

Silicon interstitials also have low formation energies near the surface. The lowest energy structure costs only $0.6 \mathrm{eV}$, when referenced to the perfect surface and the bulk Si chemical potential. In this structure, shown in Fig. 2, the Si interstitial occupies a hexagonal site between the second and third layers of the surface. The calculated formation energies of vacancies and interstitials are given as a function of depth below the surface in Figs. 3 and 4, respectively. ${ }^{30}$ It is seen that the dominant native point defects at and near the surface are, in decreasing order of concentration, surface addimers, surface divacancies, the near-surface hexagonal interstitial, vacancies in the top two layers and surface adatoms. The minimum energy needed to form a Frenkel pair near the surface, namely a vacancy and an interstitial, is $1.4 \mathrm{eV}$, substantially lower than the value of $7.2 \mathrm{eV}$ in the bulk. This bears out the generally prevalent assumption that surfaces are sources and sinks of vacancies and interstitials.

In summary, this section makes it clear that there are a number of novel structural configurations of native point defects at and near the $\mathrm{Si}(100)$ surface with greatly reduced formation energies relative to the bulk material, Therefore, concentrations of defects are significant even at moderate temperatures. ${ }^{8,9}$ These defects can thus assist impurity incorporation and migration at temperatures much lower than those at which bulk diffusion becomes feasible. While reduc-

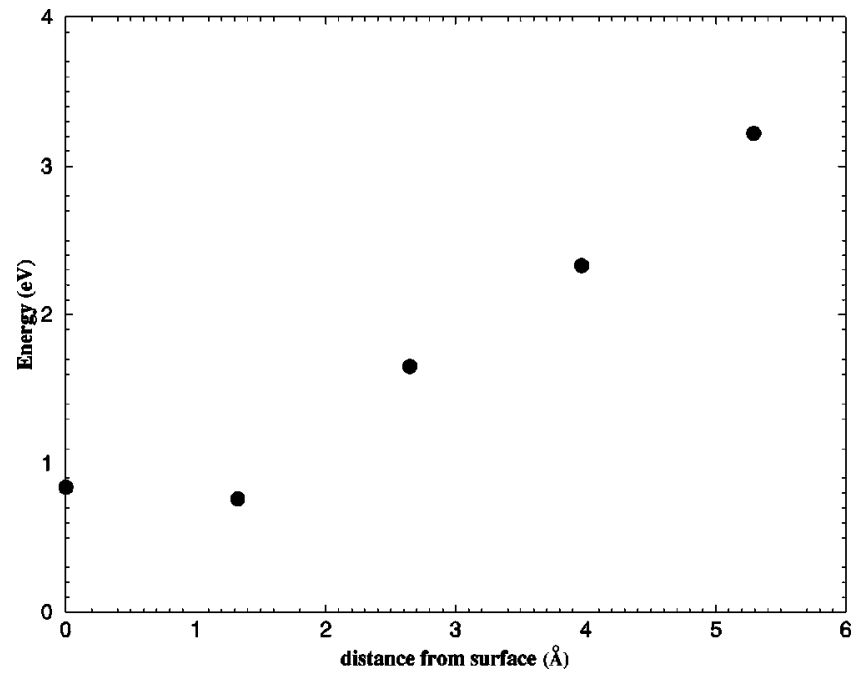

FIG. 3. Vacancy formation energies as a function of depth below the $\mathrm{Si}(100)$ surface.

tion in defect energies in the first or second layer of the surface might have been expected, we have found that it persists to even deeper subsurface layers. Thus, in a significant region near the surface, defect dynamics differs considerably from the bulk. It should be possible to exploit this result in the engineering of nanoscale devices. ${ }^{28}$

\section{ADSORPTION OF ARSENIC ATOMS}

We now move on to a description of the interaction of As atoms with the $(2 \times 1) \mathrm{Si}(100)$ surface. A number of configurations of impurity adatoms and addimers were investigated in order to determine the energetically most-favorable structures. The equilibrium geometries of the As adatom and addimer are similar to those of the $\mathrm{Si}$ adatom and addimer. In the lowest configuration, the As addimer is on top of a dimer row, bonded to two neighboring Si dimers. In this case, configurations parallel and perpendicular to the surface $\mathrm{Si}$ dimers differ in energy by $0.5 \mathrm{eV}$, with latter being the lowest in energy. Configurations with the As addimer positioned

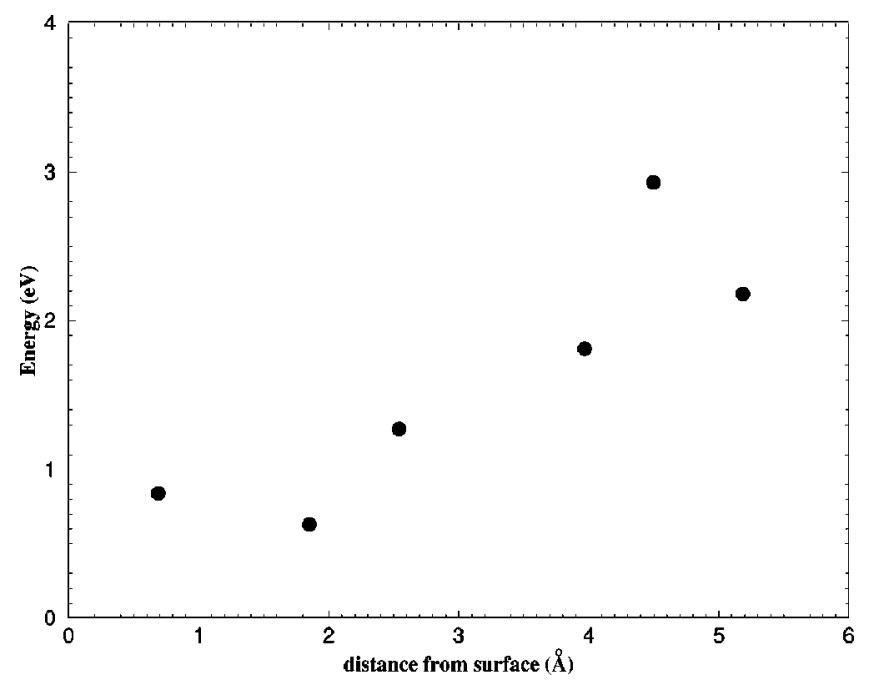

FIG. 4. Si interstitial formation energies as a function of depth below the $\mathrm{Si}(100)$ surface. 
in the trenches between dimer rows are over $1.0 \mathrm{eV}$ higher. It has been found both experimentally ${ }^{1}$ and theoretically ${ }^{29,30}$ that it is thermodynamically favorable for an adsorbed As dimer to incorporate into the $\mathrm{Si}(100)$ surface, displacing a surface Si dimer. The substitutional As impurities at the surface can then diffuse into the material with the assistance of near-surface vacancies and interstitials. Our calculations show that the substitutional configuration in the surface layer is favored by $0.9 \mathrm{eV}$ with respect to the second layer, and $1.25 \mathrm{eV}$ with respect to the third layer of the surface. It is clear that As atoms have a strong preference for the first layer, and thus it is energetically favorable for the incorporated As atoms to completely occupy all available surface sites, giving rise to a surface terminated by a ( 2 $\times 1$ ) reconstruction formed by As dimer rows.

\section{DEFECTS AT THE ARSENIC-TERMINATED SURFACE}

The $\mathrm{Si}(100)$ : As surface has a $(2 \times 1)$ reconstruction and is covered by rows of As dimers. Each As atom has five valence electrons, of which three participate in chemical bonds with neighboring atoms while the other two form a lone pair. Therefore, this surface has no unpaired electrons (i.e., dangling bonds), unlike the clean $\mathrm{Si}(100)$ surface. We found that the maximum binding energy of an $\mathrm{As}_{2}$ molecule to the $\mathrm{Si}(100)$ :As surface is $0.60 \mathrm{eV}$, much lower than the $4.31 \mathrm{eV}$ value on the clean surface. This is consistent with the experimental observations ${ }^{5}$ of low reactivity of the $\mathrm{Si}(100)$ :As surface to gaseous atoms and molecules. As expected, the chemical passivation of the surface is correlated with its low chemical reactivity.

Uhrberg et al., ${ }^{2}$ making use of the results of systematic angle-resolved photoemission experiments and $a b$ initio pseudopotential calculations, showed that the electron energy bands of the two surfaces are completely different. Recently, Kipp et al. reported results ${ }^{7}$ on the electronic properties of the $\mathrm{Si}(100)$ and $\mathrm{Si}(100)$ :As surfaces using reflectance difference spectroscopy, scanning tunneling microscopy (STM), and theoretical calculations, which show distinct signatures of the dangling bond states at the Si-Si dimers in the former and the As-As dimers in the latter. Therefore, the electronic structure of the two surfaces are completely different.

We have found that the chemical passivation induced upon As termination drastically alters the properties of surface and subsurface point defects. At the clean surface, the reduction of defect formation energies of vacancies was shown to be associated with large atomic relaxations of the Si neighbors. This resulted in significant chemical rebonding, which, in turn, healed the defects to a considerable extent. The chemical bonds at the $\mathrm{Si}(100)$ :As surface, on the other hand, are very rigid and the atomic relaxations in the vicinity of vacancies are very small. In Figs. 5(a)-5(c) we show the relaxed structures of the surface divacancy and the vacancies in the first and the second layers, respectively, of $\mathrm{Si}(100)$ :As. The atoms neighboring the vacancies are close to their positions near the perfect surface. Therefore, there is little chemical rebonding of Si neighbors of the vacancies, resulting in high-formation energies of the defects. Indeed, the calculated formation energies of surface As vacancies and divacancies at the $\mathrm{Si}(100)$ :As surface are 4.1 and $6.0 \mathrm{eV}$, respectively. ${ }^{31}$ (a)

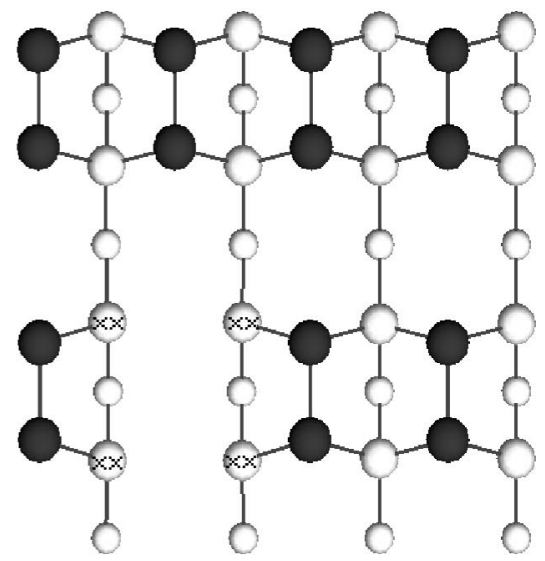

(b)

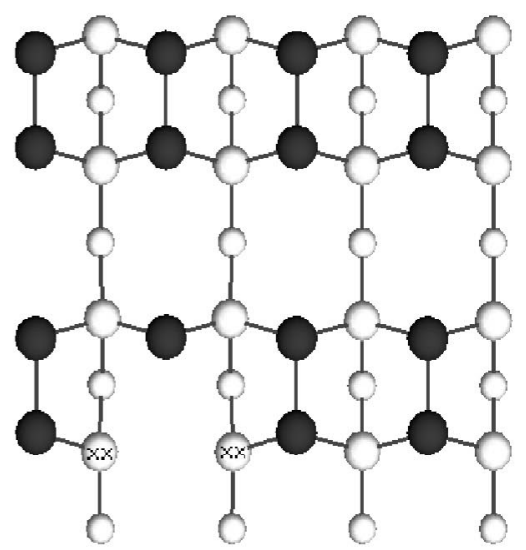

(c)

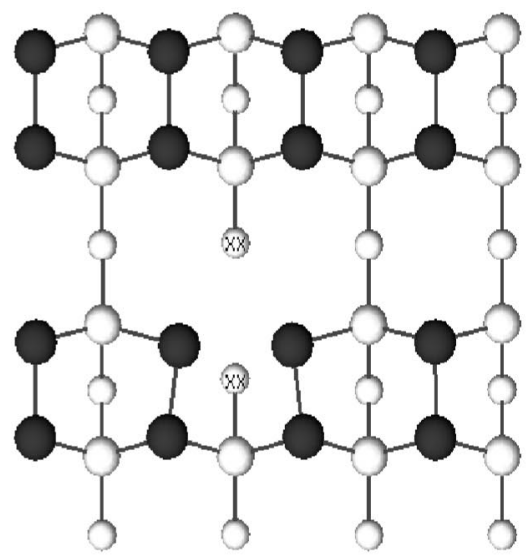

FIG. 5. Top view of the relaxed structures of vacancies at the $\mathrm{Si}(100)$ :As surface. The Si neighbors of the vacancies are marked with " $x x$." (a) surface divacancy; (b) first layer vacancy; (c) second layer vacancy.

Silicon vacancies in the subsurface region have formation energies greater than $3.0 \mathrm{eV}$.

The reduced reactivity of the surface also increases the energies of the near-surface interstitials. For instance, the formation energy of the $\mathrm{Si}$ interstitial at $\mathrm{Si}(100)$ : As in the configuration similar to that of Fig. 2 for $\mathrm{Si}(100)$ is $1.9 \mathrm{eV}$. Its structure is shown in Fig. 6. While this is significantly higher than the 0.6 value for the clean surface, it is still much lower than the formation energies for near-surface vacancies that are plotted in Fig. 7. Several other configurations of interstitial Si were explored near the $\mathrm{Si}(100)$ :As surface and found to have formation energies in the range $2.0-3.0 \mathrm{eV}$. 


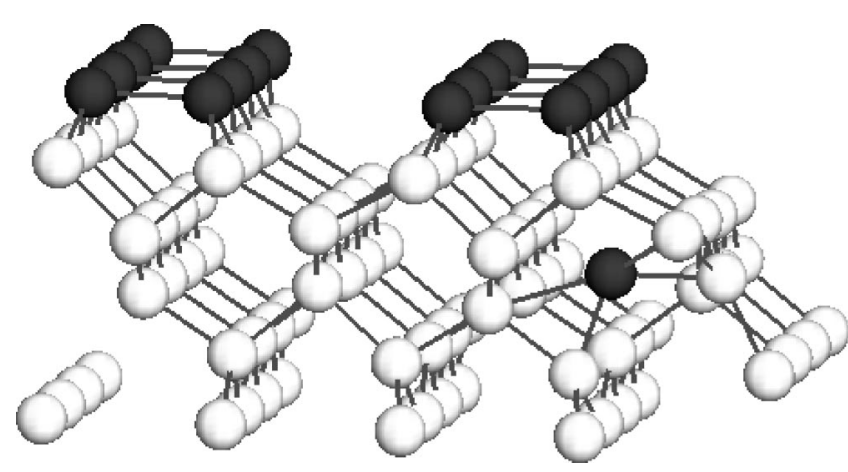

FIG. 6. Side view of the relaxed structure of the lowest energy $\mathrm{Si}$ interstitial configuration near the $\mathrm{Si}(100)$ :As surface. The interstitial is shaded for clarity.

These results show that the chemical passivation induced upon the formation of the surface As monolayer drastically alters the properties of surface and subsurface point defects. The high formation energies of vacancies near the $\mathrm{Si}(100)$ :As surface explain the experimental observations $\mathrm{s}^{2,5,7}$ of highly ordered, essentially defect-free rows of As dimers. This is in contrast to the clean $\mathrm{Si}(100)$ surface, which always has a certain fraction of surface divacancies, irrespective of the mode of surface preparation. ${ }^{8,9}$

The asymmetry between the energetics of vacancies and interstitials near the $\mathrm{Si}(100)$ :As surface is striking. In bulk Si, the formation energies of vacancies and interstitials are comparable, being in the range of 3.3-3.9 eV. ${ }^{12,32}$ At the clean $\mathrm{Si}(100)$ surface this near-equivalence is preserved, while the magnitude of the energies is in the neighborhood of $1.0 \mathrm{eV}$. However, at the $\mathrm{Si}(100)$ :As surface, surface and subsurface vacancies require significantly more energy to form than interstitials. Therefore, the concentration of vacancies at any given surface temperature will be much lower than that of interstitials. In the following section, this will be shown to have important implications for the nature of impurity incorporation and migration near this surface.

\section{IMPURITY INCORPORATION INTO THE BULK}

We proceed to apply the above results to the phenomenon of diffusive incorporation of adsorbed impurities into the

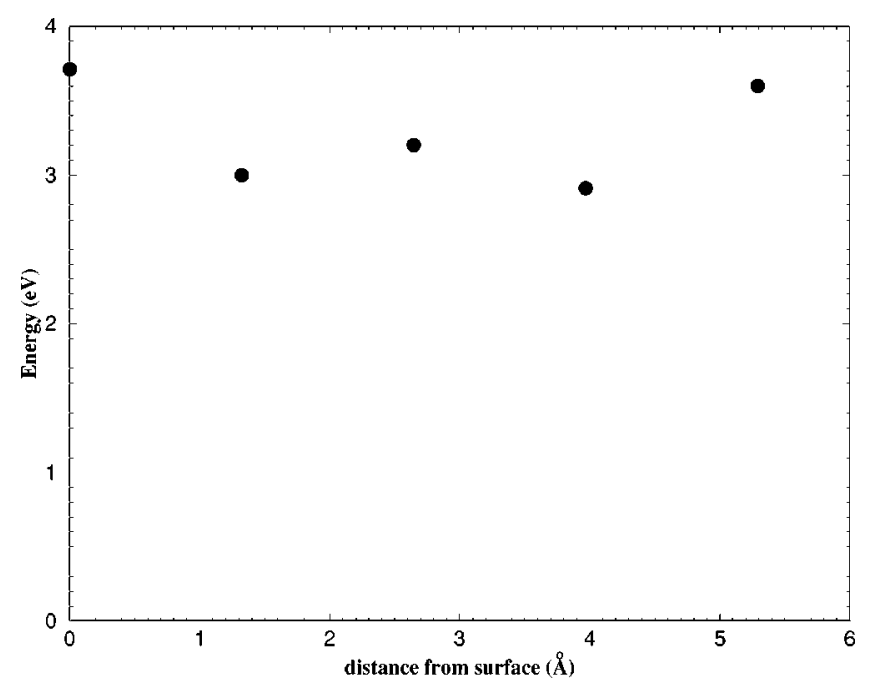

FIG. 7. Vacancy formation energies as a function of depth below the $\mathrm{Si}(100)$ : As surface. bulk. In an earlier publication, ${ }^{30}$ we had presented a detailed discussion of the various pathways for the initial stages of As and $\mathrm{P}$ incorporation into the clean $\mathrm{Si}(100)$ surface. It was argued that the diffusion of an impurity addimer into a surface divacancy is the preferred pathway at moderate incorporation temperatures. This is consistent with a number of experimental observations. ${ }^{1,20}$ In this section, we address the process of incorporation of As impurities into the bulk once the surface is completely terminated with As dimers.

Given that the $\mathrm{Si}(100)$ :As surface is already covered by As atoms, the simplest incorporation step would require the migration of the As adatom into a stable subsurface position. In a vacancy-assisted incorporation mechanism, an impurity adatom in proximity to a subsurface vacancy would migrate into the site occupied by the vacancy. The high-formation energies of subsurface vacancies, of the order of $3.0 \mathrm{eV}$, and the further energy of about $0.5 \mathrm{eV}$ required for vacancy hopping ${ }^{16}$ would imply a lower bound of $3.5 \mathrm{eV}$ for the activation energy of the vacancy mechanism. This is close to the activation energy of As diffusion in bulk $\mathrm{Si}$, which is negligible below a temperature of about $800^{\circ} \mathrm{C} .{ }^{16}$ Therefore, a vacancy-assisted mode of incorporation would require similar incorporation temperatures.

Turning to interstitial incorporation mechanisms, we examined two distinct pathways: (i) The direct motion of an As adatom into a subsurface interstitial position through open channels of the $\mathrm{Si}(100)$ :As surface. The interstitial As atom knocks out a subsurface $\mathrm{Si}$ atom ("kick-out process",12,13) and takes up a substitutional position in a subsurface layer. Subsequently, the interstitial Si atom diffuses away from the As atom or the two diffuse into the bulk as a defect complex. (ii) The As adatom kicks out a $\mathrm{Si}$ atom exposed to the surface in the second or third layer. Again, the thus created $\mathrm{Si}$ interstitial can either diffuse away and be annihilated at the surface or migrate into the bulk with the As atom. Both mechanisms lead to the As atom entering a substitutional position in the subsurface region and the ejection of an $\mathrm{Si}$ atom as an adatom or a subsurface interstitial.

In order to investigate the As adatom incorporation pathways, several configurations of As adatoms were examined to determine its equilibrium structure. The lowest energy structure corresponds to the As atom being in a trench between the As dimer rows, bonded to two surface As atoms on two sides of the trench and subsurface $\mathrm{Si}$ atoms. This configuration is illustrated in Fig. 8(a). Another structure, with the As atom in a configuration similar to the equilibrium $\mathrm{Si}$ adatom ${ }^{19}$ is only $0.3 \mathrm{eV}$ higher in energy. We then investigated the energetics of the process of As incorporation via diffusion in the interstitial channel. The structures shown in Figs. 8(b) and 8(c) had energies of about 2.0 and $1.5 \mathrm{eV}$ above the starting structure. In these calculations, the incorporating As atom was moved in small steps of about $0.1 \AA$ in the appropriate direction. All the other atoms in the simulation cell were relaxed at every step. The subsequent kick out of an $\mathrm{Si}$ atom to make the As atom incorporate into the lattice required only about $1.0 \mathrm{eV}$, which is comparable to the result for bulk Si. ${ }^{13,33}$ The maximum energy of the system for this incorporation mechanism, denoted by (i) in the previous paragraph, was only about $2.5 \mathrm{eV}$. We carried out a similar simulation for the mechanism labeled (ii) above and obtained an energy barrier of a similar magnitude. 
(a)

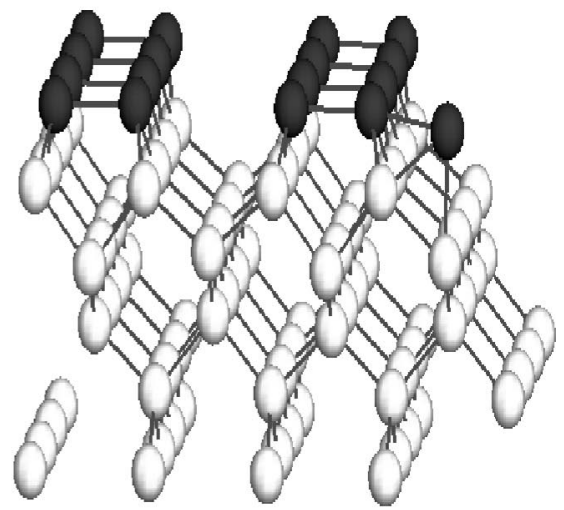

(b)

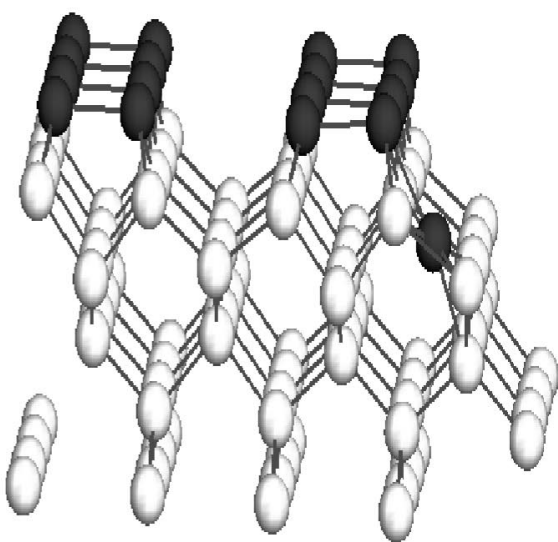

(c)

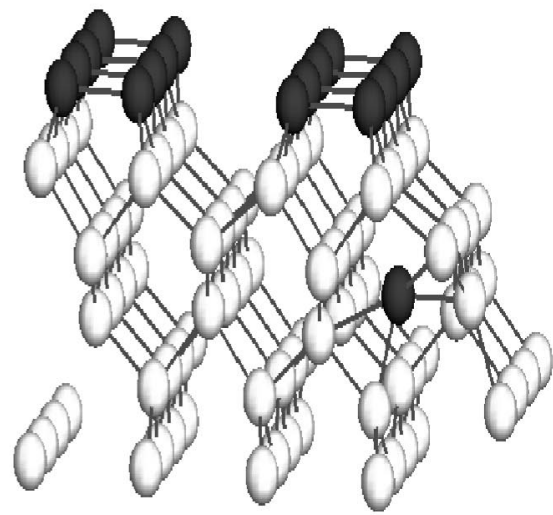

FIG. 8. Schematic view of the interstitial mechanism of incorporation of an As adatom into $\mathrm{Si}(100)$ :As. (a) The lowest energy configuration of the As adatom; (b)-(c) intermediate steps of As incorporation into the bulk. See text.

Therefore, the incorporation of adsorbed As with the assistance of silicon interstitials should dominate over vacancy-assisted pathways at moderate diffusion temperatures. One should also note that the activation energy barriers of $2.5 \mathrm{eV}$ quoted here are only upper bounds. An exhaustive search of the potential energy surface of this system might reveal pathways, which have even lower activation energies. It is also important to note that the calculated activation energies are significantly lower than those required for As migration in bulk $\mathrm{Si}$, namely $4.0 \mathrm{eV} .{ }^{12}$ For nanoscale devices, this presents the possibility for impurity incorporation at lower temperatures than those needed for engineering conventional silicon chips.

Turning to experiments, thus far, to the best of our knowl- edge, the experimental activation energy for As incorporation by in-diffusion into the $\mathrm{Si}(100)$ surface has not been reported. However, an important consequence of the interstitial mechanisms of incorporation is the injection of $\mathrm{Si}$ interstitials into the material. At low surface doping levels and incorporation rates, each $\mathrm{Si}$ interstitial that is generated is likely to be rapidly annihilated at the surface. However, at high doping levels, a strong flux of Si interstitial atoms may lead to their trapping in the bulk. Indeed, agglomerates of interstitials formed as a result of diffusive As incorporation have been detected as dislocation loops upon thermal annealing of the doped material. ${ }^{14,34}$ The density of these loops is found to increase with dopant concentration and with decreasing processing temperatures. We propose that some fraction of the observed dislocation loops forms upon agglomeration of $\mathrm{Si}$ interstitials generated in the material during diffusive incorporation of As. On the other hand, a fraction of these interstitials might also have been generated in the bulk during thermal annealing, via the kickout of $\mathrm{Si}$ atoms neighboring substitutional As impurities, as was proposed by Rousseau et $a l^{35}$ to explain their observations of annealed Si films implanted with high doses of As ions. However, Shibayama et $a l^{36}$ observed enhanced diffusion of As atoms in heavily As-doped samples obtained via diffusive incorporation of As, with rates that were many orders of magnitude higher than those that are typically observed at their diffusion temperature. They also found that B impurities that were present in a deeper layer below the Asincorporated region also exhibited similarly enhanced diffusion. It is well known ${ }^{12,13,33}$ that boron diffusion is predominantly assisted by Si interstitials. Therefore, the observations of Shibayama et al. can be explained by the influx of a large number of $\mathrm{Si}$ interstitials during the incorporation process, as we described above.

\section{SUMMARY AND CONCLUSIONS}

In summary, we have shown that the structures and energies of point defects at the clean and the arsenic-passivated $\mathrm{Si}(100)$ surfaces are totally different. The passivation of surface dangling bonds is found to dramatically alter the structural properties of the atomic layers near the surface. The clean $\mathrm{Si}(100)$ surface, which has a high density of surface dangling bonds, is very flexible and permits large relaxations in the vicinity of defects. The $\mathrm{Si}(100)$ :As surface, on the other hand, is free of dangling bonds and inhibits atomic rearrangements around defects. The difference between the properties of these two surfaces is strictly of a chemical nature, because $\mathrm{Si}$ and As atoms have similar atomic sizes and thus strain effects are negligible. ${ }^{5}$

Near the clean $\mathrm{Si}(100)$ surface, both vacancies and interstitials have low formation energies, less than $1.0 \mathrm{eV}$, far lower than the values in the neighborhood of $3.5 \mathrm{eV}$ found in the bulk. The reasons for this reduction are rather transparent for near-surface vacancies and interstitials, extensive atomic relaxations, and chemical rebonding take place in the vicinity of the defects. Both interstitials and vacancies have comparably low formation energies near the surface and should be readily available at moderate temperatures to mediate impurity incorporation and migration. This is consistent with experiments that show that adsorbed As atoms incorporate into the surface layer at temperatures in the neighborhood of $400{ }^{\circ} \mathrm{C} .{ }^{1}$ 
However, the $\mathrm{Si}(100)$ :As surface has a completely different response to the formation of defects. At this surface, atomic relaxations near vacancies are negligible and the neighboring atoms remain close to their ideal bulklike positions. The undercoordination of these atoms raises the formation energies of these defects to high values in the range of 3.0-4.0 eV, similar to those found in the bulk. ${ }^{12,32,16,33}$ While the formation energies of near-surface interstitials rise by over $1.0 \mathrm{eV}$ upon As passivation, they are still about 2.0 $\mathrm{eV}$, much lower than the bulk value of $3.3 \mathrm{eV} .^{32}$ Therefore, the equilibrium concentration of $\mathrm{Si}$ interstitials would be much higher than that of vacancies at the $\mathrm{Si}(100)$ : As surface. This favors an interstitial mechanism for the diffusive incorporation of adsorbed As atoms into the bulk, which leads to the generation of Si interstitials in the bulk material.

The preponderance of the interstitial mechanism relative to the vacancy mechanism has important consequences for heavily As-doped Si. This is because As is well known to form electrically inactive complexes with vacancies at rather moderate temperatures, above a critical doping level. ${ }^{15,16}$ Since As passivation of the surface suppresses the formation of vacancies relative to interstitials, our results explain why it is possible to incorporate large amounts of electrically ac- tive As atoms into the bulk via diffusion at high temperatures. It is only upon thermal annealing that vacancy generation or migration into the material gives rise to deactivation of a major fraction of incorporated As impurities. ${ }^{14,34,16}$

In conclusion, a monolayer of arsenic atoms on the $\mathrm{Si}(100)$ surface drastically affects both the electronic and structural properties of the near-surface region. The formation energies of vacancies at the arsenic-terminated surface are greatly increased compared to those of the clean surface. The formation energies of Si interstitials near this surface are significantly lower than those of vacancies but still substantially greater than at the clean surface. This explains the phenomenal uniformity and stability of the $\mathrm{Si}(100)$ :As surface. ${ }^{5}$ An interstitial mechanism of diffusive As incorporation is favored, causing the generation of Si interstitials in the bulk, especially at high doping levels. The inhibition of a vacancy mechanism near the surface explains the high level of electrical activation of in-diffused As atoms.

\section{ACKNOWLEDGMENTS}

This work was supported by U.S. DOE. The calculatons were carried out at DOD and NC super computer centers.
${ }^{1}$ R. D. Bringans, D. K. Biegelsen, and L. E. Swartz, Phys. Rev. B 44, 3054 (1991). See references therein.

${ }^{2}$ R. I. G. Uhrberg, R. D. Bringans, R. Z. Bachrach, and J. E. Northrup, Phys. Rev. Lett. 56, 520 (1986).

${ }^{3}$ M. Copel, M. C. Reuter, E. Kaxiras, and R. M. Tromp, Phys. Rev. Lett. 63, 632 (1989).

${ }^{4}$ M. Copel, M. C. Reuter, M. Horn von Hoegen, and R. M. Tromp, Phys. Rev. B 42, 11682 (1990).

${ }^{5}$ R. D. Bringans, Crit. Rev. Solid State Mater. Sci. 17, 353 (1992).

${ }^{6}$ Y. J. Ko, J. Y. Yi, S. J. Park, E. H. Lee, and K. J. Chang, Phys. Rev. Lett. 76, 3160 (1996). See references therein.

${ }^{7}$ L. Kipp, D. K. Biegelsen, J. E. Northrup, L. E. Swartz, and R. D. Bringans, Phys. Rev. Lett. 76, 2810 (1996).

${ }^{8}$ R. M. Tromp, R. J. Hamers, and J. E. Demuth, Phys. Rev. Lett. 55, 1303 (1985).

${ }^{9}$ Y. W. Mo, J. Kleiner, M. B. Webb, and M. G. Lagally, Phys. Rev. Lett. 66, 1998 (1991).

${ }^{10}$ Y. J. Chabal and K. Raghavachari, Phys. Rev. Lett. 54, 1055 (1985).

${ }^{11}$ R. D. Bringans and M. A. Olmstead, Phys. Rev. B 39, 12985 (1989).

${ }^{12}$ P. M. Fahey, P. B. Griffith, and J. D. Plummer, Rev. Mod. Phys. 61, 289 (1989).

${ }^{13}$ C. S. Nichols, C. G. Van de Walle, and S. T. Pantelides, Phys. Rev. B 40, 5484 (1989).

${ }^{14}$ R. O. Schwenker, E. S. Pan, and R. F. Lever, J. Appl. Phys. 42, 3195 (1971).

${ }^{15}$ K. C. Pandey, A. Erbil, G. S. Cargill III, R. F. Boehme, and D. Vanderbilt, Phys. Rev. Lett. 61, 1282 (1988).

${ }^{16}$ M. Ramamoorthy and S. T. Pantelides, Phys. Rev. Lett. 76, 4753 (1996). See references therein.

${ }^{17}$ A. Lietoila, J. F. Gibbons, T. J. Magee, J. Peng, and J. D. Hong, Appl. Phys. Lett. 36, 765 (1980).
${ }^{18}$ D. Nobili, A. Carabelas, G. Celotti, and S. Solmi, J. Electrochem. Soc. 130, 922 (1983).

${ }^{19}$ G. Brocks, P. J. Kelly, and R. Car, Surf. Sci. 269-270, 860 (1991).

${ }^{20}$ J. Wang, T. A. Arias, and J. D. Joannopoulos, Phys. Rev. B 47, 10497 (1993).

${ }^{21}$ G. Brocks and P. J. Kelly, Phys. Rev. Lett. 76, 2362 (1996). See references therein.

${ }^{22}$ R. M. Tromp and M. Mankos, Phys. Rev. Lett. 81, 1050 (1998).

${ }^{23}$ W. Kohn and L. J. Sham, Phys. Rev. 140, A1133 (1965).

${ }^{24}$ D. R. Hamann, M. Schluter, and C. Chiang, Phys. Rev. Lett. 43, 1494 (1979).

${ }^{25}$ E. L. Briggs, D. J. Sullivan, and J. Bernholc, Phys. Rev. B 52, R5471 (1995); 54, 14362 (1996).

${ }^{26}$ D. J. Chadi, Phys. Rev. Lett. 43, 43 (1979).

${ }^{27}$ Here and in the following, the formation energies of Si defects were computed using the bulk Si chemical potential, which was calculated with a 216-atom cell.

${ }^{28}$ J. Tersoff, Phys. Rev. Lett. 74, 5080 (1995).

${ }^{29}$ B. D. Yu and A. Oshiyama, Phys. Rev. Lett. 71, 585 (1993).

${ }^{30}$ M. Ramamoorthy, E. L. Briggs, and J. Bernholc, Phys. Rev. Lett. 81, 1642 (1998). Some of the formation energies of near-surface defects were reported briefly in this publication.

${ }^{31}$ The formation energies of As vacancies and divacancies were computed using as references the perfect $\mathrm{Si}(100)$ :As and the equilibrium As addimer configuration on $\mathrm{Si}(100)$ :As.

${ }^{32}$ P. E. Blochl, E. Smargiassi, R. Car, D. B. Laks, W. Andreoni, and S. T. Pantelides, Phys. Rev. Lett. 70, 2435 (1993).

${ }^{33}$ J. Zhu, T. D. dela Rubia, L. H. Yang, C. Mailhiot, and G. H. Gilmer, Phys. Rev. B 54, 4741 (1996).

${ }^{34}$ S. Hirota, M. Miyake, S. Nakayama, and E. Arai, J. Electrochem. Soc. 137, 318 (1990).

${ }^{35}$ P. M. Rousseau, P. B. Griffin, and J. D. Plummer, Appl. Phys. Lett. 65, 578 (1994).

${ }^{36}$ H. Shibayama, H. Masaki, and H. Hashimoto, Appl. Phys. Lett. 27, 230 (1975). 